\title{
Android Application Design for Automatic Tablet Dispenser
}

\author{
Mrs. Husna Tajwar \\ Dept of Electronics and Instrumentation \\ P.D.A College of Engineering \\ Kalaburagi Karnataka India
}

\author{
Prof. Veena Saraf \\ Assistant Professor, Dept of E\&C \\ P.D.A. College of Engineering \\ Kalaburagi Karnataka Indi
}

\begin{abstract}
This project patient medication reminder is a system which helps in medication administration and monitoring. This system consists of an ATMEGA328P microcontroller with an inbuilt EEPROM and a real-time circuit. This system is driven by an embedded program that inputs predefined parameters which are processed based on the input variables entered via a user interface device such as the keypad. All the entries made on the keypad are concurrently and simultaneously displayed on embedded program to initiate the alert through an audio alarm. Not only does it have an alarm system, but also it notify through IoT to user the medicine to be taken at the reminder time.
\end{abstract}

\section{INTRODUCTION}

The motivating force behind this design is based on the desire to alleviate the problem faced by the aged and physically challenged people in trying to monitor patients in hospitals or at home. It also takes into consideration the disabled or aged people that may have problems to remind themselves of the time and the medicine to be taken. A substantial number of patients, particularly the handicapped and the elderly do not follow instructions in taking medication. This can result in a patient failing to take medication, taking the wrong medication, taking an incorrect amount of medication, or taking the medication at a wrong time, leading to either a drug overdose or an ineffective application of medication to the patient. The elderly are especially prone to problems since they often take several medications and have failing memories. Care takers such as nurses also need to be reminded on occasion that a patient needs to take a particular medication at a predetermined time (Rita M. Agans, 1992). There is a recognized need for providing medicines on a regularized basis with timed notice to the person requiring them.

Patient medication reminder is useful to all patients. Nowadays, patient monitoring is a critical task. The physician has to monitor the patient's health continuously, and the prescribed medicine has to be given from time to time. There are instances when patients remember to take medicines at the stipulated time but forget which pill has to be taken. The task is to design a patient medication reminder circuit system that records the time and the name of the medicine to be taken by patients at a correct time. The time and medicine names are changed according to the patients need through the keypad connected.

\section{PROPOSED METHOD}

Our setup consists of pill dispensing kit which dispenses the medicine or pill at an appropriate time. It consists of a real-time clock which keeps track of accurate time and it is already programmed to prescribed time for dispensing. Our setup includes three sessions named morning, afternoon and night. $4 * 4 \mathrm{Keypad}$ input is used to set the current time and date. Date and time will be displayed in the $16^{*} 2 \mathrm{LCD}$ display. Once the time is set there is no need to change again and again because the real-time clock will automatically track the time and date.

Arduino is programmed in such a way that, when the current time matches with the time which is already set in real time clock it sends the control to the audio amplifier where the alarm gets activated and gives a voice note of which session it is. The alarm rings till the user or patient presses the button. If the patient presses the button, automatically the pill for the corresponding session comes out.

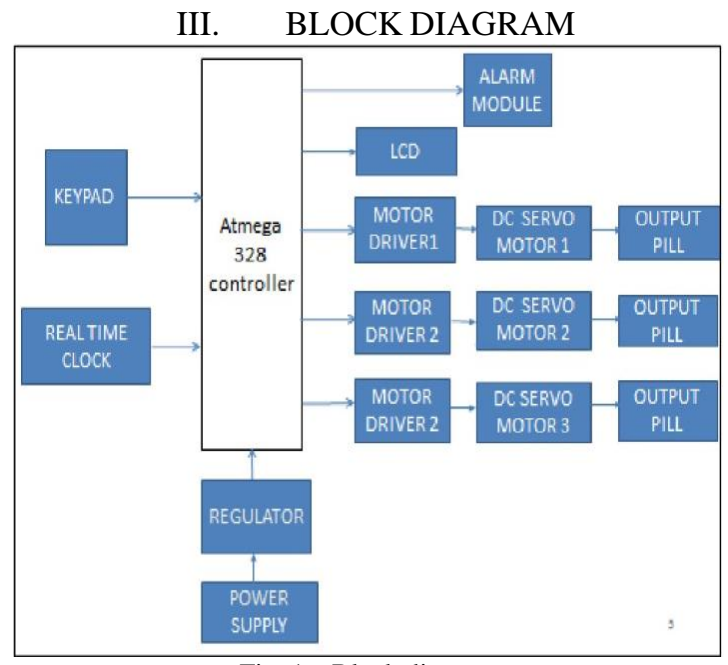

Fig. 1. Block diagram

It consists of Arduino ATMEGA328, keypad, Real Time Clock, Power supply, Motor Driver, De gear motor, Liquid crystal Display, Buzzer. Block diagram is shown in the Fig

\section{METHODOLOGY}

The micro controller ARDUINO MEGA 2560 is used to interface components such as RTC, Ultrasonic sensor, IR sensor, L293D motor driver, fingerprint sensor and GSM module, and also is used to enter and store data about the patient using a keypad present in robot. The robot waits in its home position till the time arrives to deliver the medicines to the patient in their respective room. Using real time clock RTC module the microcontroller checks if the time has arrived and once the time arrives the microcontroller selects the path in which the robot has to 
travel using the data entered. The robot uses a line follower technique to travel where the path is represented by a black line. The IR sensor in the robot send a light signal and receives back the signal only when there is a black line by this method the robot is made to move only in the black line path. After the path is selected by the microcontroller the IR sensor detects the black line and sends information to the microcontroller which will make the robot to move in the selected path with the help of the motor driven by the motor driver. There is an ultrasonic sensor present in the robot for obstacle detection, by generating a sound wave which will be reflected back only when there is a obstacle in the path. In such cases the ultrasonic sensor sends signal to the microcontroller which will stop the movement. After it reaches the patients room the system voices over the patient ID or patient name from the DF mini player using speaker. Now the system waits for fingerprint authentication. The patient or the caretaker places their finger on the fingerprint scanner. The optical fingerprint scanner compares the fingerprint with the data and if it matches, it gives the authentication to the microcontroller which opens the pill box using the servo motor. In the LED display it displays the medicine and its quantity. After the patient has taken the medicine the push button is pressed which will close the pill box. The robot uses a GSM module to send information whether the patient has taken their medicines or not to the registered mobile number. The number may be the patient's relatives or guardian.

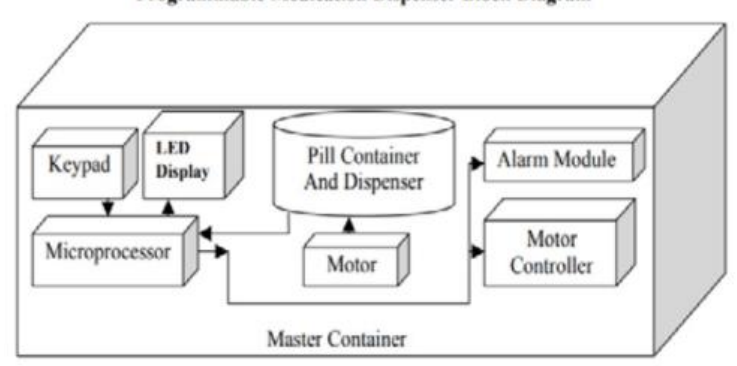

The robot closes the pill box and uses the same line following technique to return to its home position.

\section{RESULT}

We made our project as useful for the patient who needs this and all related users. We conclude result that our project is useful for those people who are taking pills regularly, prescription of medicine is very long and hard to remember for those users. Our product is so useful that it can cure those patients illness and there will no need of taking care of these types of patients so caregiver has no tension about their health and they will live healthy and tension free life.

The working of the system is explained:

Step1 : Switching on the device.

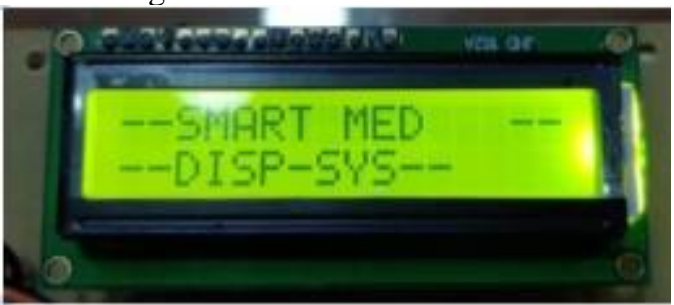

Step 2: The time is set when the system needs to dispense the medicine and also the fingerprint is scanned.
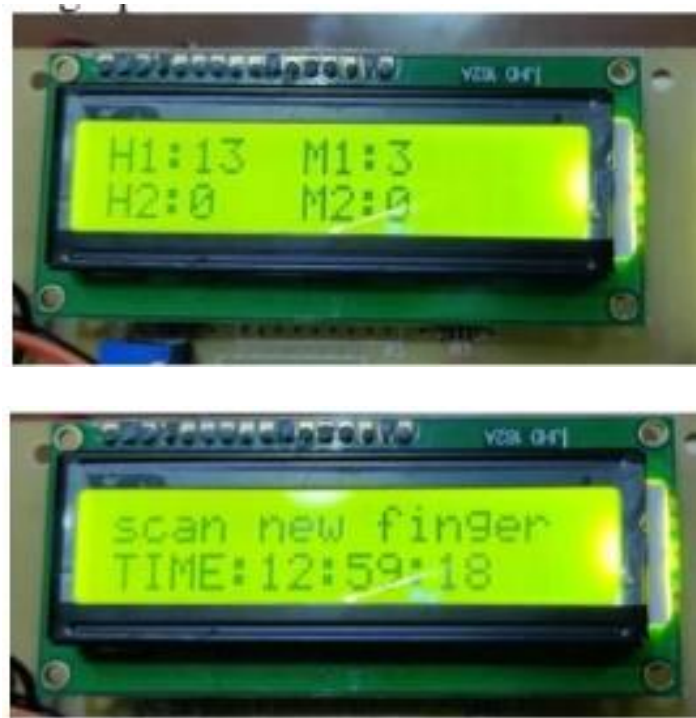

Step 3: At the particular time, the system starts moving from its home position and reaches the patient's room. Once the authorized fingerprint is kept, the servo motor dispenses the medicine.

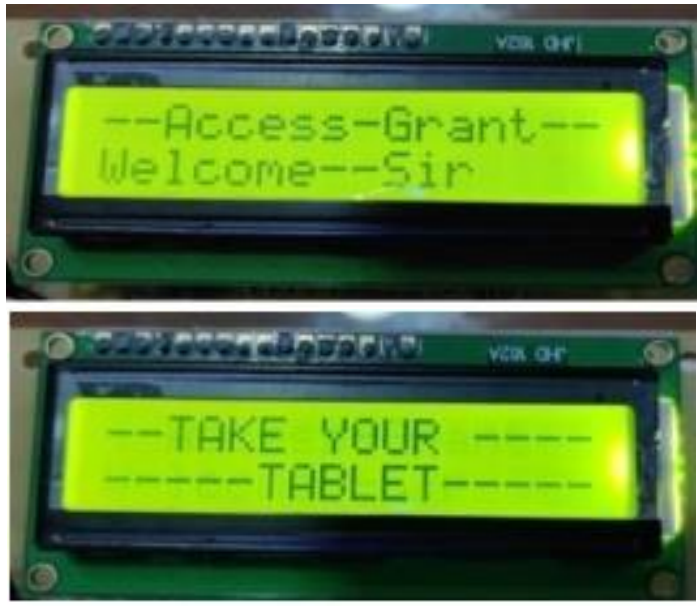

Step 4: A Notification indicating whether the patient has taken the medicine is being sent to the registered mobile number.

\section{$09: 38$}

The PATIENT has taken the tablet.

The PATIENT hasn't taken the tablet. 


\section{CONCLUSION}

In this project, an efficient and portable dispensing is designed with low-cost and simple electronics. The medicines are filled once in a month. The outcome of our project is that buzzer notifies the person by alarm to intake the pills or capsules on time. It reminds the patient and indicates what session it is. Hence the corresponding tablet will come out into the tray. In future this project can be implemented by using GSM module. Even if they are indicated by alarm to take their respective pills, the user may forget to take their pill. Through GSM module, the message will send to corresponding caretaker about the medicine which the users have not taken at scheduled time.

\section{ACKNOWLEDGMENT}

I am thankful to my guide, Prof. Veena Saraf for her constant encouragement, guidance and for the continues support in making this work complete.

\section{REFERENCES}

[1]. Viral Shah, Jigar Shah, Nilesh Singhal, Harsh Shah \& Prof. Prashant Uapdhyay, "Smart Medicine Box", Imperial Journal of Interdisciplinary Research (IJIR), Vol-2, Issue-5 ,2016.

[2]. Naga Udayini Nyapathi1, Bhargavi Pendlimarri2, Karishma Sk3 , Kavya Ch4," Smart Medicine Box using ARM 7 Micro controller", International Research Journal of Engineering and Technology(IRJET), Volume: 03 Issue: 05 | May-2016.

[3]. Aakash Sunil Salgia*, K. Ganesan and Ashwin Raghunath, "Smart Pill Box", Indian Journal of Science and Technology, Vol 8(S2), 189-194, January 2015.

[4]. P. Raga Lavima1, Mr. G. Subhramanya Sarma2, "AN IOT BASED INTELLIGENT MEDICINE BOX”, IJCSMC, Vol. 4, Issue. 10, October 2015, pg.186 - 191 .

[5]. Suneetha Uppala1, B. Rama Murthy2, Smart Medicine Time Indication Box, International Journal of Science and Research (IJSR), Volume 6 Issue 1, January 2017.

[6]. Aakash Sunil Salgia*, K. Ganesan and Ashwin Raghunath(January 2015), Smart Pill Box, US2009/0299522 A1.

[7]. Bo pi, Halton pi (May 5, 2016), Smart pill container, control method and system, US 20160120758 A1. 